\title{
Unfavorable hydroxychloroquine COVID-19 research associated with authors having a history of political party donations
}

\author{
Andrew C. Berry ${ }^{1, *}$, Russell S. Connering ${ }^{2}$, Ivan Rodriguez ${ }^{3}$, Qianying Zhang ${ }^{4}$, Bruce B. Berry ${ }^{5}$ \\ ${ }^{1}$ Division of Gastroenterology, Larkin Community Hospital, South Miami, 33143 FL, USA \\ ${ }^{2}$ Department of Ophthalmology and Visual Sciences, The Medical College of Wisconsin, Milwaukee, 53226 WI, USA \\ ${ }^{3}$ Department of Accounting and Finance, Gary M. Owen College of Business, Eastern Michigan University, Ypsilanti, 48197 MI, USA \\ ${ }^{4}$ Department of Economics and Business Administration, Hillsdale College, Hillsdale, 49242 MI, USA \\ ${ }^{5}$ Community Physicians, Froedtert and the Medical College of Wisconsin, Milwaukee, 53226 WI, USA \\ *Correspondence: Aberry5555@gmail.com (Andrew C. Berry) \\ DOI:10.31083/j.rcm.2021.01.262 \\ This is an open access article under the CC BY 4.0 license (https://creativecommons.org/licenses/by/4.0/). \\ Submitted: 27 November 2020 Revised: 20 December 2020 Accepted: 24 January 2021 Published: 30 March 2021
}

We explored the degree to which political bias in medicine and study authors could explain the stark variation in Hydroxychloroquine (HCQ)/Chloroquine (CQ) study favorability in the US compared to the rest of the world. COVID-19/SARS-CoV-2 preprint and published papers between January 1, 2020-July 26, 2020 with Hydroxychloroquine and/or Chloroquine; 267 met study criteria, 68 from the US. A control subset was selected. HCQ/CQ study result favorability (favorable, unfavorable, or neutral) was noted. First and last main authors of each US study were entered into FollowTheMoney.org Website, extracting any history of political party donation. Of all US studies (68 total), 39/68 (57.4\%) were unfavorable, with only $7 / 68$ (10.3\%) of US studies yielding favorable results-compared to 199 non-US studies, 66/199 (33.2\%) unfavorable, 69/199 (34.7\%) favorable, and 64/199 (32.2\%) neutral. Studies with at least one US main author were $20.4 \%$ (SE 0.053, $P<0.05$ ) more likely to report unfavorable results than non-US studies. US Studies with at least one main author donating to any political party were $25.6 \%$ (SE $0.085, P<0.01$ ) more likely to have unfavorable results. US studies with at least one author donating to the Democratic party were $20.4 \%$ (SE 0.045, P $<0.05$ ) more likely to have unfavorable results. US authors were more likely to publish studies with medically harmful conclusions than non-US authors. Cardiology-specific HCQ/CQ studies were $44.2 \%$ more likely to yield harmful conclusions $(P<0.01)$. Inaccurate propagation of $\mathrm{HCQ} / \mathrm{CQ}$ cardiac adverse effects with individual scientific author political bias has contributed to unfavorable US HCQ/CQ publication patterns and political polarization of the medications.

\section{Keywords}

COVID-19; SARS-CoV-2; Hydroxychloroquine; Chloroquine; Political party; Donations; Political bias; Cardiac

\section{Introduction}

"Unfortunately, the political climate that has persisted has made any objective discussion about this drug impossible, and we are deeply saddened by this turn of events. Our goal as scientists has solely been to report validated findings and allow the science to speak for itself, regardless of political considerations. To that end, we have made the heartfelt decision to have no further comment about this outside the medical community-staying focused on our core mission in the interest of our patients, our community, and our commitment to clinical and academic integrity" (Henry Ford Health System) [1].

SARS-CoV-2 (COVID-19) has been known to the world since December 31, 2019, and on March 11, 2020 the World Health Organization (WHO) declared it a pandemic [2]. The seriousness and rapidity of this novel disease introduced intense uncertainty. As early as the end of February 2020, it was recognized that little was known about this novel virus and what was known would be rapidly changing [3]. Epidemiologic models were used early in the disease and instilled a sense of urgency [4]. Numerous clinical trials of all forms have been designed or completed on potential therapeutic medical regimens for COVID-19, mainly Hydroxychloroquine (HCQ), Chloroquine (CQ), Remdesivir, and Dexamethasone-with no worldwide consensus for best practice. HCQ has carried a political designation ever since US President Donald Trump and government leaders touted the drug on March 19, 2020, saying it could "have a chance to be one of the biggest game changers in the history of medicine [5]". On March 28, 2020, the FDA issued Emergency Use Authorization (EUA) for HCQ and CQ in treatment for hospitalized COVID-19 patients [6]. Amid political strain and varying results, on June 15, 2020, the FDA rescinded its EUA [7]. Significant political strain has dampened its investigation potential and even prevented physicians from prescribing it in fear of political, social, and litigation pressures $[8,9]$. The clash between medical science and politics has reached a level unforeseen in prior times. Since the pandemic, publishing on COVID-19 has taken on a torrid pace, and is increasingly accessed openly by the general public, not just scientists [10]. In times of crisis, it is absolutely essential that transparency 
of information and freedom from bias be insured so that informed decisions can be made by patients in the choices in their healthcare.

"Just as a functional, trusting, and transparent collaboration between physicians and their patients is necessary for informed consent, a trusting relationship must exist between our governmental health officials and the public. Efforts to suppress direct communication are detrimental to this goal" (Collins) [11]. Furthermore, "these officials must remain free of political bias so that their message is a transparent communication of facts"(Collins) [11].

It is not the purpose of this paper to articulate an argument for or against, the medical use of HCQ/CQ, but rather to explore the possibility of political bias influencing the design and outcome of HCQ/CQ scientific research being used to formulate public opinions. The amount of negative press for HCQ/CQ in the US seemed out of proportion for a medication used by millions of people worldwide for numerous common medical diseases for decades, intensely studied, and with minimal adverse effect profile. Globally, initial studies supported HCQ/CQ use and were socially accepted by those populations as helpful. But in the US, some of the same studies were discredited, shunned, and handled negatively by the media. As objective scientists, we became concerned if the uncertainties of the disease, its seriousness, intense politicization of treatment, and the change in patterns of publication could have influenced the design and outcome of the studies or the inherent timeline of study result favorability [12]. The aim of our study was to explore the degree to which political bias in medicine could explain the variation in HCQ/CQ study favorability in the US compared to the rest of the world. We choose to utilize US main author political party donation history as a surrogate measure of possible author political bias. Has investigation in medicine lost its objectively and succumbed to external political pressure and introduced political bias into findings?

\section{Methods}

We utilized publicly-available online German-based natural language processing software, Collabovid (https://ww w.collabovid.org/) [13], to electronically search all COVID19/SARS-CoV-2 preprint and published papers from main preprint servers (medRxiv, BioRxiv, arXiv) and noted peer-reviewed publication servers (Elsevier, PubMed). All searched papers had either of the keywords COVID-19 or SARS-CoV-2 in their abstract or title. We searched between January 1, 2020 and July 26, 2020. A total of 27,028 listings were noted. We then searched using the words HYDROXYCHLOROQUINE or CHLOROQUINE, and 312 listings were noted. Of these, 45 were excluded for varying predetermined reasons: retracted studies (2), case reports (10), clinical trial methods publication (9), unrelated/other (14). Additionally, (10) of the 312 listings were preprints later published in full peer-reviewed form, with the preprint version now excluded.

Study result favorability was recorded by one study author
Table 1. Study Demographics

\begin{tabular}{|c|c|c|}
\hline & HCQ/CQ Studies & COVID-19 Controls \\
\hline & $\mathrm{n}=267$ & $\mathrm{n}=68$ \\
\hline HCQ & $153(57.3)$ & . \\
\hline CQ & $38(14.2)$ & . \\
\hline Both & $76(28.5)$ & . \\
\hline Favorable & $76(28.5)$ & . \\
\hline Unfavorable & $105(39.3)$ & . \\
\hline Neutral & $86(32.2)$ & . \\
\hline Preprint & $71(26.6)$ & $30(44.1)$ \\
\hline Elesevir & $63(23.6)$ & $13(19.1)$ \\
\hline PubMed & $133(49.8)$ & $25(36.8)$ \\
\hline RCT & $11(4.1)$ & $0(0.0)$ \\
\hline Prospective & $26(9.7)$ & $6(8.8)$ \\
\hline Retrospective & $45(16.9)$ & $33(48.5)$ \\
\hline Systematic Review & $36(13.5)$ & $0(0.0)$ \\
\hline Basic Science & $52(19.5)$ & $0(0.0)$ \\
\hline Review/Editorial & $97(36.3)$ & $29(42.6)$ \\
\hline Impact Factor & 5.0 & 3.6 \\
\hline Altimetric Score & 549.6 & 41.4 \\
\hline Cardiac & $38(14.2)$ & . \\
\hline Patient Involvement & $146(54.7)$ & . \\
\hline Pharmacy-Based & $37(13.9)$ & . \\
\hline
\end{tabular}

Legend: CQ, Chloroquine; HCQ, Hydroxychloroquine; RCT, Randomized Controlled Trial.

(A.C.B.) by reviewing the abstract and discussion/conclusion sections of the full manuscript. Unfavorable studies coincided with a negative conclusion based on the particular study hypothesis. For example, HCQ/CQ may have provided "no benefit, no decrease in hospitalization, no decrease in mortality, no prophylaxis benefit, harm to the patient in any manner, lack of positive benefit, etc”. Favorability coincided with a positive study conclusion based on the particular study hypothesis and the medication in question, HCQ and/or CQ. Any study with neutral conclusions was noted as neutral. Any study with ambiguous conclusions was noted as neutral. To note, all article favorability extraction was performed before any search into author political party donation history. In order to gauge differences in severity of the favorability findings above, we tested the proportion of studies that concluded HCQ/CQ was medically harmful from US vs. non-US based authors.

First and last author demographics were extracted, as these authors represent the lead author and article guarantor for the content of the article, referred to as the main authors throughout this article. Subsequently, any US-based main author was manually searched by one study author (A.C.B.) on publicly-available Website FollowTheMoney.org (https: //www.followthemoney.org/) [14]. If a search yielded a match for the name and state, further listing description was evaluated for the donor's professional title or workplace. Only then, after all checkpoints for full verification that the author was indeed the match for the listed donor, was it counted as such. Political party of the donation was noted as Democratic, 
Table 2. Donation Political Party for US Studies

\begin{tabular}{lccccc}
\hline & Democratic (\%) & Republican (\%) & Other (\%) & Non-Donors & Num. Authors \\
\hline HCQ/CQ & $15(88.2)$ & $2(11.8)$ & $0(0.0)$ & 108 & 125 \\
COVID-19 Controls & $8(40.0)$ & $8(40.0)$ & $4(20.0)$ & 116 & 136 \\
\hline
\end{tabular}

Legend: Total donors includes unique first and last main authors only.

Table 3. HCQ/CQ vs. COVID-19 Control Political Party Donations

\begin{tabular}{lcccc}
\multicolumn{5}{c}{ Political Party Donations } \\
\hline & HCQ/CQ COVID-19 & Difference & $P$-Value \\
& Studies & Controls & & \\
\hline Democrat & 0.120 & 0.059 & 0.061 & $0.041^{a}$ \\
Republican & 0.016 & 0.059 & -0.043 & $0.036^{a}$ \\
Non-donors & 0.864 & 0.858 & 0.011 & 0.400 \\
Amount (\$) & 159.496 & 67.081 & 92.415 & 0.100 \\
\# Donations & 0.296 & 0.191 & 0.105 & 0.177 \\
Authors ${ }^{b}$ & 125 & 136 & & \\
Total Studies & 68 & 68 & & \\
\hline
\end{tabular}

Legend: Difference in means test. For $P$-values, one-tail test used to determine significance, ${ }^{a} P<0.05$. ${ }^{b}$ Authors: Encompasses first and last (main) author totals only.

Republican, Other. If no match was noted or in question, it was deemed no donation was made by the author.

To serve as a control subset for COVID-19 publication trends and author characteristics, we randomly selected 68 control studies pertaining to COVID-19 but not involving HCQ/CQ from the same time period. We searched Collabovid with the filter UNITED STATES, yielding 810 studies (from the 27,028 total). A random sampling technique was used, and if the study matched a prior reason for exclusion or if there was only one author, it was not selected. The same study characteristics were recorded and the same process used to assess political party donation history.

Linear regression was used to measure the association between study conclusion favorability and the authors' location (US vs. non-US) for our global sample and to measure the association between study conclusion favorability and donation history for our US subsample. Additional variables are included in the regression to control for study characteristics that could also influence conclusion favorability. Twosided $P$-values calculated with the $t$-test for the ordinary least squares coefficient estimate are reported, with $P<0.05$ considered statistically significant. Additional measures using Boschloo's test to better control for small-sample/rare-event bias were performed. Data analysis was performed with Stata version 15.0 (StataCorp LP, College Station, TX, USA). No participants or patients were involved.

\section{Results \\ 3.1 Demographics and controls}

There were $267 \mathrm{HCQ} / \mathrm{CQ}$ papers extracted, of which, 68 had the first or last author affiliated with a US-based institution. Subsequently, 68 COVID-19 non-HCQ/CQ control studies were randomly extracted from a time-matched inter- val. Demographic data can be seen in Table 1.

Of the 68 US HCQ/CQ papers, 17 total main authors were donors: 15 (88.2\%) Democratic, 2 (11.8\%) Republican, and 0 Other Party. Of the time-matched 68 COVID-19 nonHCQ/CQ COVID-19 only controls, there were 20 total main author donors: 8 (40\%) Democratic, 8 (40\%) Republican, and 4 (20\%) Other Party (Table 2). Difference of means test resulted in statistically significant more Democratic donors (6.1\%, $P<0.05)$ and fewer Republican donors $(4.3 \%, P<$ 0.05) in the HCQ/CQ group as compared to the COVID-19 control donor sample (Table 3 ).

\subsection{Study geographic location and HCQ/CQ study favorability and harm}

Table 4 shows US versus non-US HCQ/CQ study favorability totals. Most notably, 39 (57.4\%) of US studies were unfavorable, with only 7 (10.3\%) of US studies yielding favorable results. Compared to Non-US studies with 66 (33.2\%) unfavorable, 69 (34.7\%) favorable, and 64 (32.2\%) neutral. Multivariate regression analysis with study characteristics as controls is seen in Appendix 1. Studies with at least one US main author were 20.4\% (SE 0.053, $P<0.05$ ) more likely to report unfavorable results than non-US studies. Subsequently, US main author studies were also 19.6\% (SE 0.024, $P<0.01)$ less likely to report favorable results than non-US studies.

In order to better gauge differences in severity of the findings above, studies with conclusions noting medical harm were analyzed. Out of a total of 534 authors, 63/409 nonUS authors and 41/125 US authors were part of studies with harmful conclusions. US-authored studies had a much higher proportion of studies yielding harmful conclusions than nonUS studies $(32.8 \%$ vs. $15.4 \%, P<0.001, t$-test and Boschloo test) (Table 5).

A logistic regression analysis for all HCQ/CQ studies with harmful conclusions is shown in Appendix 2, noting differences in proportions when controlling for other study characteristics. There remained a correlation between harmful studies and US authors even after regression analysis (0.094, SE 0.060), but it was not statistically significant $(P>0.05)$. Notably, studies of cardiology nature (or subject) had the strongest significant correlation to harmful HCQ/CQ studies overall $(0.456$, SE $0.085, P$-value: $<0.01)$.

\subsection{Donor, political party affiliation, and study favorability}

US Studies with at least one main author donating to any political party were $25.6 \%$ (SE $0.085, P<0.01$ ) more likely to have unfavorable results and $20.1 \%$ (SE 0.080, $P<0.05$ ) less likely to have neutral results. US studies with at least one author donating to the Democratic party were $20.4 \%$ (SE 
Table 4. HCQ/CQ Study Favorability for

US vs. Non-US Studies

\begin{tabular}{lcc}
\hline & US (\%) & Non-US (\%) \\
\hline Unfavorable & $39(57.4)$ & $66(33.2)$ \\
Favorable & $7(10.3)$ & $69(34.7)$ \\
Neutral & $22(32.4)$ & $64(32.2)$ \\
Total & 68 & 199 \\
\hline
\end{tabular}

Table 5. Difference in Proportion of a Conclusion of Harm Between US and non-US Studies

\begin{tabular}{ccccc}
\hline \multirow{2}{*}{ US (\%) } & \multirow{2}{*}{ Non-US (\%) } & Difference (\%) & \multicolumn{2}{c}{$P$-values } \\
\cline { 3 - 5 } & & & $t$-test & Boschloo \\
\hline Harmful 41/125 (32.8) & $63 / 409(15.4)$ & 17.4 & $0.001^{a}$ & $0.001^{a}$ \\
\hline
\end{tabular}

Legend: Out of a total of 534 authors, 63/409 non-US authors and 41/125

US authors were main authors of studies with harmful conclusions.

${ }^{a} P<0.001$

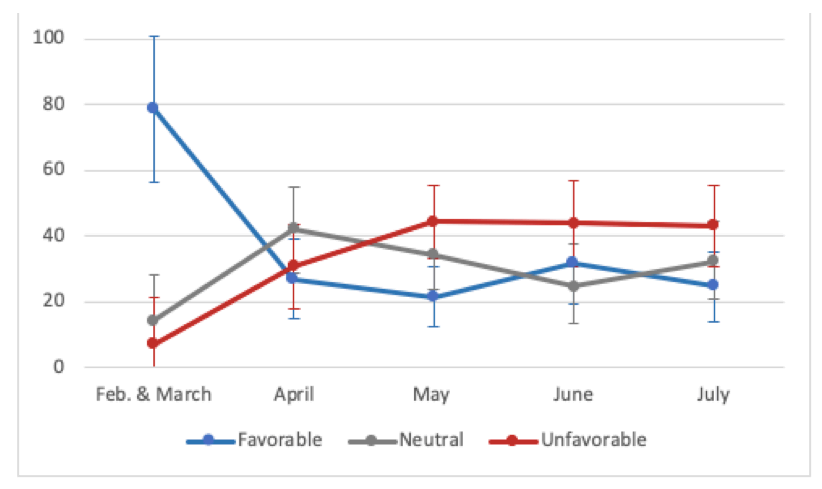

Fig. 1. HCQ/CQ Study Release Timeline and Study Favorability. Legend: All studies are for year 2020. July is until July 26, 2020 only.

0.045, $P<0.05)$ more likely to have unfavorable results and $17.0 \%$ (SE 0.094, $P<0.10$ ) less likely to report neutral results (Appendix 3, Appendix 4). Only two US studies had at least one main author donating to the Republican party, both with unfavorable results. Thus, optimal analysis could not be performed without rare-event sample bias. Appendix 3, Appendix 4 further list other multivariate regression analysis for US-based studies and study characteristics.

\subsection{Study characteristics and favorability}

Studies characterized as editorial reviews were $38.6 \%$ less likely to yield favorable results (SE 0.050, $P<0.01$ ) (Appendix 1). Furthermore, Table 6 displays US author editorial review totals and author political party donation history. Almost all the editorial reviews were unfavorable or neutral $(21 / 22,95.5 \%)$, with any listed history of donation all coming from a main author of Democratic party donation record $(7 / 7,100 \%)$.

\subsection{HCQ/CQ study release timeline and study favorability}

Table 7 depicts month-by-month HCQ/CQ study release and study favorability, with a graphical representation shown in Fig. 1. Although February/March had the fewest total
Table 6. US Editorial Reviews and Donation History

\begin{tabular}{lcccc}
\hline & $\begin{array}{c}\text { Num. Editorials } \\
(\%)\end{array}$ & $\begin{array}{c}\text { Democratic Republican } \\
(\%)\end{array}$ & $(\%)$ & $(\%)$ \\
\hline Unfavorable & $11(50.0)$ & $6(85.7)$ & 0 & 0 \\
Favorable & $1(4.5)$ & $0(0.0)$ & 0 & 0 \\
Neutral & $10(45.5)$ & $1(14.3)$ & 0 & 0 \\
Total & 22 & 7 & 0 & 0 \\
\hline
\end{tabular}

studies listed, $78.6 \%$ had results that were favorable, with only 7.1\% yielding unfavorable results. A clear shift away from studies with favorable results toward those of unfavorable results occurred starting in April, reaching a paradigm shift in May-July. The percentage of neutral studies peaked in April, with a downtrend thereafter (Fig. 1).

\subsection{Preprint to peer-reviewed publication status}

Of the 267 analyzed HCQ/CQ project interval research studies, 71 of 267 (26.6\%) were preprint studies and not yet published. Only 10 of the excluded and original 312 listings were preprints that later became published during the study period, suggesting only 10 of 81 (71 included +10 excluded) total preprints (12.3\%) went from preprint status to full peerreviewed journal publication during the study period ending July 26, 2020.

\section{Discussion 4.1 Main discussion points}

Our results describe the differences in HCQ/CQ favorability outcomes from US-based research compared to the rest of the world. SARS-CoV-2, and the chemical composition of $\mathrm{HCQ} / \mathrm{CQ}$ are constants, the political environment in which the studies were performed and authored is different. The sheer study favorability breakdown worldwide suggest a near-even split between unfavorable (33.2\%), favorable (34.7\%), and neutral (32.2\%) HCQ/CQ studies. Meanwhile, $57.4 \%$ of US studies were unfavorable, with a mere $10.3 \%$ conveying favorable results (Table 4). Even after accounting for numerous potential confounding variables, US-authored studies were significantly more likely to report unfavorable results and less likely to report favorable results. We suggest that author bias may account for some if not all of the differences. Unfortunately, the intense media exposure in this US election year has polarized our society and may be injecting various forms of bias into medical research, making the transparency termed essential by Collins et al. difficult to achieve [11].

"Transparency is a necessary condition of trust. And when trust is forfeited, so too is the opportunity to persuade people to alter behavior patterns for the common good"(Collins) [11].

Not only is there an unexplained preponderance and deviation of US study unfavorable results from worldwide HCQ/CQ studies, but among US main study authors, those who donated money to any political party were $25.6 \%$ more likely to report unfavorable results and $20.1 \%$ less likely to report neutral results (Appendix 3). This begs the question, 
Table 7. HCQ/CQ Study Release Timeline and Study Favorability

\begin{tabular}{lcccc}
\hline \multirow{2}{*}{ Month } & \multirow{2}{*}{ Study Total } & \multicolumn{2}{c}{ Mean Favorability \% $(95 \%$ CI $)$} & \multirow{2}{*}{ Neutral } \\
\cline { 3 - 4 } & & Favorable & Unfavorable & \\
\hline February/March & 14 & $78.6(56.3,100.9)$ & $7.1(-6.9,21.1)$ & $14.3(-4.7,33.3)$ \\
April & 52 & $26.9(14.8,39.1)$ & $30.8(18.1,43.4)$ & $42.3(28.8,55.9)$ \\
May & 79 & $21.5(12.4,30.6)$ & $44.3(33.3,55.3)$ & $34.2(23.7,44.7)$ \\
June & 57 & $31.6(19.4,43.8)$ & $43.9(30.9,56.9)$ & $24.6(13.3,35.8)$ \\
July & 65 & $24.6(14.1,35.2)$ & $43.0(31.0,55.2)$ & $32.3(20.9,43.8)$ \\
Total & 267 & 28.5 & 39.3 & 32.2 \\
\hline \multicolumn{2}{l}{ Legend: All studies are for year 2020. July is until July 26, 2020 only. 95\% confidence interval (CI) listed } \\
in parenthesis.
\end{tabular}

are those US authors who are passionate enough to donate money to a specific political party also sacrificing the objective principles of scientific research, and their unbiased sworn-upon Hippocratic oath to now unjustly influence scientific literature in alignment with their political beliefs? The general public recognizes the harm that bias in reporting on the COVID-19 epidemic poses to society [15], with implications of such bias to society magnified in times of crisis [16]. The surfacing of such bias during the COVID-19 pandemic even landed a full article in the Summer issue of the MIT Sloan Management Review, detailing not one, but nine different biases regarding COVID-19 decision-making [17].

We included a control arm of our study to establish baseline author political party donation patterns of those authors of COVID-19 scientific studies-not related to HCQ/CQ. An even party donation history split between Democrats and Republicans was found, with the remaining aligning to "Other" parties. However, in those HCQ/CQ studies in which an author had a history of political party donation, the vast majority $(15 / 17,88.2 \%)$ of donating authors donated to the Democratic party. Only 2 donating main authors on all HCQ/CQ papers had a history of Republican party donation. US studies with at least one author donating to the Democratic party were $20.4 \%$ (SE $0.045, P<0.05$ ) more likely to have unfavorable results and $17.0 \%$ (SE 0.094, $P<0.10$ ) less likely to report neutral results (Appendix 4). Did Democratic party donating authors choose to publish research on $\mathrm{HCQ} / \mathrm{CQ}$ intending to find negative conclusions, or did Republican authors voluntarily avoid any scientific investigation into HCQ/CQ for fear of negative results and radicalizing from their party line leadership?

The article type with the least objective methodology is an editorial review. Almost all US editorial review article types were unfavorable or neutral $(21 / 22,95.5 \%)$. Seven of those editorial authors had a history of political party donation, and all seven authors donated exclusively to the Democratic party (Table 6). Did authors who supported the Democratic party choose to disseminate a perceived reputable view to the public through this article type, or did the Republican party voluntarily choose not to discuss HCQ/CQ in such manner? Regardless, a propensity for editorial reviews skewed and polarized one direction does not facilitate unbiased and proper science. A follow-up question surfaces, did journal reviewers and editors also insert their political bias into inviting such editorial reviews or choosing which to publish? We did not seek to establish the political donation history of the journal editors, but it is conceivable that they too could be subject to political bias.

With COVID-19 came a sharp rise in rapid, open dissemination of study data via preprint screening servers, where studies are publicly-available without peer-review. Our analysis of all combined US and worldwide HCQ/CQ studies found an unpublished preprint study was $20.7 \%$ more likely to be unfavorable and $22.1 \%$ less likely to convey neutral results, holding other variables constant (Appendix 1). Approximately $25 \%$ of all studies analyzed were preprint studies. Although a dynamic and time-lagged process, a mere $12.3 \%$ of all total preprints went from preprint status to full peerreviewed journal publication during the defined study period. Submitting a preprint might have given a scientific author a modality to quickly sway public opinion using credentialed trust as a scientific research "paper". A significant portion of news media and the general public has no understanding of a preprint process. The mere lack of preprint to full publication status over this timeframe may suggest some authors had no intention of publishing in a peer-reviewed journal, knowing their study may lack such scientific merit, rather harnessing public persuasive power. With such shift towards quick and less rigorous review in scholarly communication, a sobering possibility of large entities controlling scientific thought arises. Internet-based mega-companies such as Google, Amazon, Facebook, and Apple may become interested in spearheading further transformation and outcompete current stakeholders in scholarly communication, developing more advanced user-friendly digital tools $[18,19]$. Baffy et al. [18] believe "such developments could potentially lead to a few large entities controlling the gateways to scientific knowledge, a sobering thought”. Even heavily accessed open Websites such as Wikipedia include polarizing statements such as its summary for HCQ: "Hydroxychloroquine is being studied to prevent and treat coronavirus disease 2019 (COVID-19), but all clinical trials conducted during 2020 found it is ineffective and may cause dangerous side effects. The speculative use of hydroxychloroquine 
for COVID19 threatens its availability for people with established indications" [20]. These conclusions are quite concerning, especially considering they are not authored by scientists and are designed to widely propagate through social media.

Many authors were inaccurately propagating cardiacrelated adverse effects of HCQ/CQ used in the COVID-19 patient as a means for the drug unfavorability. Studies have further shown the cardiac safety of HCQ/CQ, and any such arrythmia associated with the COVID-19 infectious process, and not the medications involved [21, 22]. Our analysis further analyzed study favorability into studies noting conclusions that were medically harmful. Our results suggest HCQ/CQ studies with US authors were more likely to express harmful conclusions than non-US authors (Table 5). A study may be unfavorable, but to be actually medically harmful comes with further bearing. We did notice that if a HCQ/CQ study involved cardiology as the main subject, these studies were 44.2\% $(P<0.01)$ more likely to yield harmful author conclusions (Appendix 2).

We performed a time-sequence analysis of monthly study favorability noting a clear demarcation from favorable HCQ/CQ studies in February/March that coincided with Trump's support for HCQ/CQ, to a shift into unfavorable studies in April through the end of the study period (Table 7, Fig. 1). A critic may suggest a reason for the favorability shift over time may just be that the medication simply does not work after more and more time for scientific investigation. Although a possibility, the sheer political polarization of this medication does not align with the scientific data, the majority coming outside the confines of the US politicallyinfiltrated border.

Our study results may suggest a means for current skeptics against $\mathrm{HCQ} / \mathrm{CQ}$ use to insert their political views into science (as evidenced by their political party donations), which not only influences the mainstream media, but may have contributed to the unparallel FDA action revoking their EUA for HCQ/CQ for COVID-19 on June 15, 2020 [7]. Around the globe, numerous well-designed studies suggest favorable results $[23,24]$ and real-life examples of successful clinical implications exist, with widespread social acceptance [25]. Politicization of medical therapies in a time of crisis is not a new concept. In 1987, AIDS was spreading fear across the world and Bactrim, one potential treatment for the deadly associated Pneumocystis Pneumonia, showed promising results. Not only did US officials not approve Bactrim for prophylaxis until two years later in 1989, but even encouraged people with AIDS to stop taking treatments, like Bactrim, that weren't specifically approved for use in people with AIDS [26].

HCQ has been in use and approved since 1955, used for an array of common diseases, with minimal side effects and with numerous current studies confirming this [27, 28]. HCQ has now taken on a political identity in the US. Censorship of articles by the very organizations about which we were warned by Baffy et al. [18] has become a reality, and the criticism of the censorship is viewed as right wing "conspiracy theory" [9]. Positive articles about HCQ in COVID-19 are labeled as "misinformation". Negative articles are labeled as "science". A bias in referencing only articles that support the author's own ideological thinking persists. Scientific journals have gone on record as supporting political candidates, Scientific American has polarized itself and has backed a presidential candidate (Biden) for the first time in 175 years, saying "This year we are compelled to do so [29]". Yet in the same issue publishing an article titled, "We can't allow the CDC [Centers for Disease Control and Prevention] to be tainted by politics [30]". Nonetheless, certain scientific authors and editors appear to be opening science to political persuasion, either hidden and inherent in the study design and conclusions, or fully transparent as The Lancet's highly unusual step of publishing a blatantly political editorial calling for Trump's ouster [31].

The Lancet was forced to recently retract an unfavorable study of HCQ/CQ in COVID-19, because the data could not be verified [32]. Verification of data, on a paper of this magnitude of importance, is expected to be done prior to publication. Could a conformational bias against HCQ/CQ have influenced the editorial staff? The Lancet's current editor, Richard Horton, in 2015, offered this insightful opinion:

"The case against science is straightforward: much of the scientific literature, perhaps half, may simply be untrue. Afflicted by studies with small sample sizes, tiny effects, invalid exploratory analyses, and flagrant conflicts of interest, together with an obsession for pursuing fashionable trends of dubious importance, science has taken a turn towards darkness" (Horton) [33].

Physicians who have reported on their own positive experience with HCQ have been censored or even fired from their positions $[8,9]$.

'Politically, it is convenient to present an alleged 'consensus' and dismiss any contrary findings as inherently illegitimate. But that action overlooks the fact that science often advances the most when presented with contradictory findings that must be resolved. If scientists routinely dismissed research findings just because they differed from previous research, science would make no progress" (Schumm) [34].

\subsection{Strengths and limitations of study}

Our study utilizes objective public-domain data openly available to all readers and with objective donation party delineations-a novel methodology removing many concerning study bias. Our inclusion of a non-HCQ/CQ COVID19 control group subset for baseline analysis of main author political party donation helps objectively assess baseline author donation characteristics, and objectively demonstrate a true deviation of author political party donation characteristics from a timeframe-matched, COVID-19-matched baseline.

Although ideal, we could not extract main author political party registration data, as not all US states allow or require voters to declare a party designation. Although the first and last author act as the most influential authors of the pa- 
per, we did not gather political party donation data for the other remaining authors, which may preclude full analysis. Although the main authors' bias would likely prevail due to academic seniority. Study favorability group classification was performed by only one senior author (A.C.B.), without any personal history of political party donations, after preextraction methodology consensus agreement by all the participating authors. Publication of HCQ/CQ studies is a very dynamic process, and we could not include studies most recent to time of this publication.

\section{Conclusions}

We have found a significant difference in the unfavorability of HCQ/CQ in US-based studies versus the rest of the world, with studies having at least one main US author significantly more likely to publish unfavorable results. US studies with a main author donating to any political party were $25.6 \%$ more likely to have unfavorable results. Furthermore, US studies with at least one author donating to the Democratic party were also $20.4 \%$ more likely to have unfavorable results.

Utilized as a potential means to propagate science swiftly and under less scrutiny, editorial reviews were a means for overall unfavorable study result propagation, and specifically for authors donating to the Democratic party. Political bias is a possible explanation for the unfavorable outcomes of the HCQ/CQ research authored in the US, influencing public and government opinions, and the subsequent non-use of the drugs here in the US. Many authors were inaccurately propagating false cardiac-related adverse effects of HCQ/CQ used in the COVID-19 patient as a means to assign unfavorability to the medication. The abrupt demarcation of study favorability with timing of political events and social pressures further illustrates the potential infiltration of politics into the once-objective scientific community. We thereby suggest the addition of "political disclosures" to the already required "financial disclosures" for scientific research submissions going forth.

\section{Author contributions}

ACB: Conceptualization, Methodology, Investigation, Data curation, Writing-Original draft preparation, WritingReviewing and Editing, Validation. RSG: Data curation, Investigation, Validation, Writing-Original draft preparation, Writing-Reviewing and Editing. IR: Investigation, Statistical analysis, Validation, Writing-Original draft preparation, Writing-Reviewing and Editing. QZ: Investigation, Statistical analysis, Validation, Writing-Original draft preparation, Writing-Reviewing and Editing. BBB: Data curation, Investigation, Validation, Writing-Original draft preparation, Writing-Reviewing and Editing.

\section{Acknowledgment}

There are no acknowledgments to disclose.

\section{Funding}

This research received no external funding.

\section{Conflict of interest}

The authors declare no financial or political conflicts of interest.

\section{Appendix}

Appendix associated with this article can be found, in the online version, at https://rcm.imrpress.com/EN/10.31083/j. rcm.2021.01.262.

\section{References}

[1] Henry Ford Health System. Hydroxychloroquine: an open letter to our community and beyond. Available at: https://www.henryfor d.com/news/2020/08/hydroxychloroquine-an-open-letter (Accessed: 19 August 2020).

[2] World Health Organization. WHO Director-General's opening remarks at the media briefing on COVID-19-11 March 2020 Available at: https://www.who.int/dg/speeches/detail/who-dir ector-general-s-opening-remarks-at-the-media-briefing-on-c ovid-19---11-march-2020 (Accessed: 25 August 2020).

[3] del Rio C, Malani PN. COVID-19-new insights on a rapidly changing epidemic. Journal of the American Medical Association. 2020; 323: 1339-1340.

[4] Holmdahl I, Buckee C. Wrong but useful-what COVID-19 epidemiologic models can and cannot tell us. New England Journal of Medicine. 2020; 383: 303-305.

[5] @realDonaldTrump. HYDROXYCHLOROQUINE \& AZITHROMYCIN, taken together, have a real chance to be one of the biggest game changers in the history of medicine. The FDA has moved mountain -Thank You! Hopefully they will BOTH ( $\mathrm{H}$ works better with $\mathrm{A}$, International Journal of Antimicrobial Agents). 2020. Available at: https: //twitter.com/realDonaldTrump/status/1241367239900778501 (Accessed: 25 August 2020).

[6] U.S. Food and Drug Administration. Letter of authorizationchloroquine phosphate and hydroxychloroquine sulfate. 2020. Available at: https://www.fda.gov/media/136534/download (Accessed: 01 September 2020).

[7] U.S. Food and Drug Administration. Coronavirus (COVID19) Update: FDA revokes emergency use authorization for chloroquine and hydroxychloroquine. 2020. Available at: https://www.fda.gov/news-events/press-announcements/coron avirus-covid-19-update-fda-revokes-emergency-use-authoriza tion-chloroquine-and (Accessed: 01 September 2020).

[8] Louise N. Dr. Simone Gold, the doctor censored by big tech over hydroxychloroquine viral video, has been fired and now lost her job. Tech Startups website. 2020. Available at: https://techstartups.com/2020/07/30/doctor-censore d-big-tech-hydroxychloroquine-viral-video-now-lost-job/ (Accessed: 30 August 2020).

[9] Herman M. Twitter blocks Dr. Samadi for hydroxychloroquine comments. Newsmax website. 2020. Available at: https://www.newsmax.com/newsmax-tv/samadi-hcq-twitter /2020/07/29/id/979655/ (Accessed: 26 August 2020).

[10] Redden E. Rush to publish risks undermining COVID-19 research. Inside Higher ED website. 2020. Available at: https://www.insidehighered.com/news/2020/06/08/fast-pac e-scientific-publishing-covid-comes-problems (Accessed: 30 August 2020).

[11] Collins KL, Markel H, Lieberman AP. Truth and transparency in a time of crisis. Journal of Clinical Investigation Insight. 2020; 5: e138132. 
[12] Barrios JM, Hochberg YV. Risk perception through the lens of politics in the time of the COVID-19 pandemic. Working Paper NO. 2020-32. 2020. Available at: https://bfi.uchicago.edu/w p-content/uploads/BFI_WP_202032.pdf (Accessed: 26 August 2020).

[13] Collabovid. Explore COVID-19 publications. 2020. Available at: https://www.collabovid.org/ (Accessed: 27 July 2020).

[14] FollowTheMoney.org. https://www.followthemoney.org/ (Accessed: 28 July 2020).

[15] Jones JM. Public sees harm in exaggerating, downplaying COVID-19 threat. Gallup poll. 2020. Available at: https://news.gallup.com/poll/298307/public-sees-harm-e xaggerating-downplaying-covid-threat.aspx (Accessed: August 2020).

[16] Jones JM. Americans see more news bias; most can't name neutral source. Gallop poll. 2018. Available at: https://news.gallup.com/ poll/225755/americans-news-bias-name-neutral-source.aspx (Accessed: 25 August 2020).

[17] Davenport TH. How to make better decisions about coronavirus. MIT Sloan management review. 2020. Avialable at: https://sloanreview.mit.edu/article/how-to-make-better-decis ions-about-coronavirus/ (Accessed: 25 August 2020).

[18] Baffy G, Burns MM, Hoffmann B, Ramani S, Sabharwal S, Borus JF, Pories S, et al. Scientific authors in a changing world of scholarly communications: what does the future hold? The American Journal of Medicine. 2020; 133: 26-31.

[19] The Social Dilemma. Directed by Jeff Orlowski. Netflix. Avialable at: https://www.netflix.com/title/81254224 (Accessed: 09 September 2020).

[20] Hydroxychloroquine. Wikipedia. Updated September 13, 2020. Avialable at: https://en.wikipedia.org/wiki/Hydroxychloroquine (Accessed: 15 September 2020).

[21] Fram G, Wang DD, Malette K, Villablanca P, Kang G, So K, et al. Cardiac complications attributed to hydroxychloroquine: a systematic review of the literature pre-COVID-19. Current Cardiology Reviews. 2020. (in press)

[22] Elsaid O, McCullough PA, Tecson KM, Williams RS, Yoon A. Ventricular fibrillation storm in coronavirus 2019. The American Journal of Cardiology. 2020; 135: 177-180.

[23] Arshad S, Kilgore P, Chaudhry ZS, Jacobsen G, Wang DD, Huitsing $\mathrm{K}$, et al. Treatment with hydroxychloroquine, azithromycin, and combination in patients hospitalized with COVID-19. International Journal of Infectious Diseases. 2020; 97: 396-403.

[24] Risch HA. Opinion: early outpatient treatment of symptomatic, high-risk COVID-19 patients that should be ramped-up immediately as key to the pandemic crisis. American Journal of Epidemiology. 2020; 189: 1218-1226.

[25] Risch HA. The key to defeating COVID-19 already exists. We need to start using it. 2020. Available at: https://www.newsweek.com/key-defeating-covid-19-alrea dy-exists-we-need-start-using-it-opinion-1519535 (Accessed: 26 August 2020).

[26] Strub S. Whitewashing AIDS history. 2017. Available at: https://www.huffpost.com/entry/whitewashing-aids-history_b _4762295?fbclid=IwAR3LHaFLzWfZtxpffdXUfcEZm-yoazjKo ZSJ87jCggwEtS6OH5TrJfnAn9Q\&guccounter=1（Accessed: 25 August 2020).

[27] Plaquenil. U.S. Food and Drug Administration. 2017. Available at: https://www.accessdata.fda.gov/drugsatfda_docs/label/2017/ 009768s037s045s047lbl.pdf (Accessed: 01 September 2020).

[28] Ruiz-Irastorza G, Khamashta MA. Hydroxychloroquine: the cornerstone of lupus therapy. Lupus. 2008; 17: 271-273.

[29] Scientific American. Scientific American endorses Joe Biden. 2020. Available at: https://www.scientificamerican.com/article/s cientific-american-endorses-joe-biden/ (Accessed: 21 September 2020).

[30] Scientific American. We can't allow the CDC to be tainted by politics. 2020. Available at: https://www.scientificamerican.com /article/we-cant-allow-the-cdc-to-be-tainted-by-politics/ (Accessed: 21 September 2020).

[31] Smith K. Trump derangement syndrome at The Lancet. National Review. 2020. Available at: https://www.nationalreview.c om/2020/06/trump-derangement-syndrome-at-the-lancet/ (Accessed: 25 August 2020).

[32] Mehra MR, Desai SS, Ruschitzka F, Patel AN. RETRACTED: hydroxychloroquine or chloroquine with or without a macrolide for treatment of COVID-19: a multinational registry analysis. The Lancet. 2020; S0140-6736(20)31180-6.

[33] Horton R. Offline: what is medicine's 5 sigma? The Lancet. 2015; 385: 1380 .

[34] Schumm WR. Navigating treacherous waters-one researcher's 40 years of experience with controversial scientific research. Comprehensive Psychology. 2015; 4. 